\title{
Effect of Repetitive Transcranial Magnetic Stimulation According to the Stimulation Site in Stroke Patients With Dysphagia
}

\author{
Jong Hwa Lee, MD, Sang Beom Kim, MD, Kyeong Woo Lee, MD, Sook Joung Lee, MD, Jae Uk Lee, MD
}

Department of Physical Medicine and Rehabilitation and Regional Cardiocerebrovascular Center, Dong-A University College of Medicine, Busan, Korea

Objective To investigate the effect of repetitive transcranial magnetic stimulation (rTMS) according to the stimulation site in subacute stroke patients with dysphagia.

Methods This study was designed as a matched comparative study. Twenty-four patients who had dysphagia after ischemic stroke were recruited, and they were divided into two groups after matching for age and stroke lesion. The patients in group A received rTMS over the brain cortex where motor evoked potential (MEP) was obtained from the suprahyoid muscle. Group B received rTMS over the brain cortex where MEP was obtained from the abductor pollicis brevis muscle. rTMS was performed at $110 \%$ of MEP threshold, $10 \mathrm{~Hz}$ frequency for 10 seconds, and then repeated every minute for 10 minutes. Dysphagia status was measured by the Functional Dysphagia Scale (FDS), the Penetration-Aspiration Scale (PAS), and the Dysphagia Outcome and Severity Scale (DOSS) using the results of a videofluoroscopic swallowing study. These evaluations were measured before, immediately, and 4 weeks after rTMS.

Results Group A showed significant improvement compared to group B in the DOSS score immediately and 4 weeks after rTMS. There were no significant differences in the changes of FDS and PAS scores between groups A and B immediately and 4 weeks after rTMS.

Conclusion rTMS over a hot spot for the suprahyoid muscle caused more improvement in swallowing function when compared to that over the interconnected site.

Keywords Transcranial Magnetic Stimulation, Stroke, Deglutition Disorders

Received August 11, 2014; Accepted October 27, 2014

Corresponding author: Jae Uk Lee

Department of Physical Medicine and Rehabilitation, Dong-A University Hospital, 26 Daesingongwon-ro, Seo-gu, Busan 602-715, Korea

Tel: +82-51-240-5691, Fax: +82-51-254-8511, E-mail: bobo-0416@hanmail.net

(c) This is an open-access article distributed under the terms of the Creative Commons Attribution Non-Commercial License (http://creativecommons. org/licenses/by-nc/4.0) which permits unrestricted noncommercial use, distribution, and reproduction in any medium, provided the original work is properly cited.

Copyright $\odot 2015$ by Korean Academy of Rehabilitation Medicine

\section{INTRODUCTION}

Dysphagia is a major complication that occurs in $37 \%$ $78 \%$ of stroke patients [1]. Typical complications of dysphagia include dehydration and malnutrition, aspiration pneumonia, and asphyxia, which can lead to death. Therefore, proper treatment after early diagnosis is critical [2-5]. Current dysphagia treatment goals include 
preventing aspiration through changes in food and water protocols, compensatory techniques, and rehabilitative exercise [6]. Reorganization in the swallowing cortical area occurs after a stroke, which is known to be related to recovery from dysphagia.

Noninvasive brain stimulation techniques are expected to change brain plasticity during recovery from poststroke dysphagia [7]. Studies using repetitive transcranial magnetic stimulation (rTMS) and transcranial direct current stimulation reported the role of noninvasive brain stimulation techniques in dysphagia recovery. Recent studies confirmed the positive effects of rTMS in treating dysphagia [8]. Khedr et al. [9] and Khedr and Abo-Elfetoh [10] applied rTMS over the esophageal cortical area of the affected hemisphere in acute dysphagic stroke patients for 2 months, and improvements in dysphagia and motor disability were observed. Verin and Lerio [11] applied rTMS over the mylohyoid cortical area of the healthy hemisphere in patients with chronic post-stroke dysphagia and detected improvements in swallowing coordination and a decrease in the aspiration score for liquids. In previous studies, rTMS was performed mostly over the cortical lesions of the swallowing muscle, from which improvements of dysphagia were observed.

However, an increasing number of functional magnetic resonance imaging (fMRI) studies have implicated multiple regions of the cerebral cortex and subcortex in the process of swallowing [12-15]. Bestmann et al. [16] showed that right-hand finger tapping led to pronounced activation in bilateral $\mathrm{M} 1 / \mathrm{S} 1$ and supplementary motor area (SMA) lesion, which are also activated during in voluntary swallowing. This indicates that there is a possibility of interconnection between both cortical areas. In a review of studies on transcranial magnetic stimulation (TMS) and fMRI, Reithler et al. [17] showed that TMS over a particular cortical site not only results in focal effects but also affects other brain regions. Furthermore, there is evidence that rTMS modulates remote areas in actual clinical settings. Triggs et al. [18] performed rTMS over the left prefrontal area and observed clinical improvements in depressed mood; they concluded that left prefrontal rTMS is capable of modulating not only the corticospinal motor system but also the orbitofrontal limbic structures, i.e., brain activities in remote areas.

A number of studies indicate that rTMS is a good treatment option for post-stroke patients. However, to the best of our knowledge, none of the studies have compared the clinical effects of rTMS applied directly over the swallowing-related cortex and the interconnected sites in poststroke dysphagia patients.

Hence, in this study, we categorized subacute stroke patients with dysphagia who underwent rTMS over specific sites of dysphagia and the interconnected site by stimulation of the cortex representing the motor hand area. Treatments were administered, after which their efficacies were compared.

\section{MATERIALS AND METHODS}

\section{Subjects}

This was a comparative study using age- and stroke lesion-matched patients. This study included subacute stroke patients with dysphagia who were admitted to our hospital between January 2012 and July 2013. The patients were divided into two groups according to the stimulation site: group A underwent stimulation of the cortex representing the suprahyoid muscle of the affected side, while group B underwent stimulation of the cortex representing the abductor pollicis brevis (APB) of the affected side. The inclusion criteria were 1) dysphagia after subacute ischemic stroke and 2) disease localized to a unilateral cerebral hemisphere (as documented by computed tomography or MRI). Exclusion criteria were 1) cerebral hemorrhage disease (arteriovenous malformation, intracortical hemorrhage, or subarachnoid hemorrhage); 2) bilateral cerebrovascular disease; 3 ) prior history of stroke; 4) swallowing difficulties unrelated to stroke; 5) inability to undergo a videofluoroscopic swallowing study (VFSS) assessment due to lack of cooperation; 6) contraindication for rTMS (seizure history, implanted pacemaker or medication pump, metal plate in the skull, or metal objects in the eye or skull); and 7) craniectomy state.

The study protocol was approved by the Institutional Review Board of Dong-A University Hospital, and all participants provided written informed consent.

\section{Intervention}

Before the rTMS, motor evoked potentials (MEPs) were evaluated using a MagPro (MagVenture Inc., Farum, Denmark). Subjects were seated comfortably in an armchair. The point of intersection between the midsagittal 
line that connects the nasion and inion and the interaural line was designated as $\mathrm{Cz}$. Cz was designated the origin (0, 0 ); the interaural line was designated the $\mathrm{x}$-axis; and the midsagittal line was designated the y-axis. Fabric, with markings at $2.5-\mathrm{cm}$ increments with $\mathrm{Cz}$ at the center, was fixed to the scalp. Magnetic stimulation was performed using a figure-of-eight coil (diameter, $2 \mathrm{~mm} \times 96 \mathrm{~mm}$ ). The target muscle in group A was the suprahyoid muscle of the affected side, while that in group B was the APB muscle of the affected side. Similar to previous studies $[19,20]$, for the suprahyoid muscle, an active electrode was attached $2 \mathrm{~cm}$ lateral to the midline of the lower chin and the reference electrode was attached to the mandibular angle (Fig. 1). The motor threshold was defined as the minimal stimulus intensity required to produce MEP $>50 \mu \mathrm{V}$ peak-to-peak amplitude in five of ten consecutive trials on each muscle. The location yielding the largest response amplitude was termed the 'hot spot', and we delivered magnetic stimulation to that point.

The magnetic instrument was used to deliver stimulatory trains using the figure-eight coil. rTMS was performed on the ipsilesional hemisphere hot spot at $110 \%$ of each MEP threshold, at $10 \mathrm{~Hz}$, for 10 seconds, and repeated every minute for 10 minutes in each group (total, 1,000 pulses). rTMS was performed once per day for 10 minutes on 10 consecutive days (five times per week for 2 weeks).
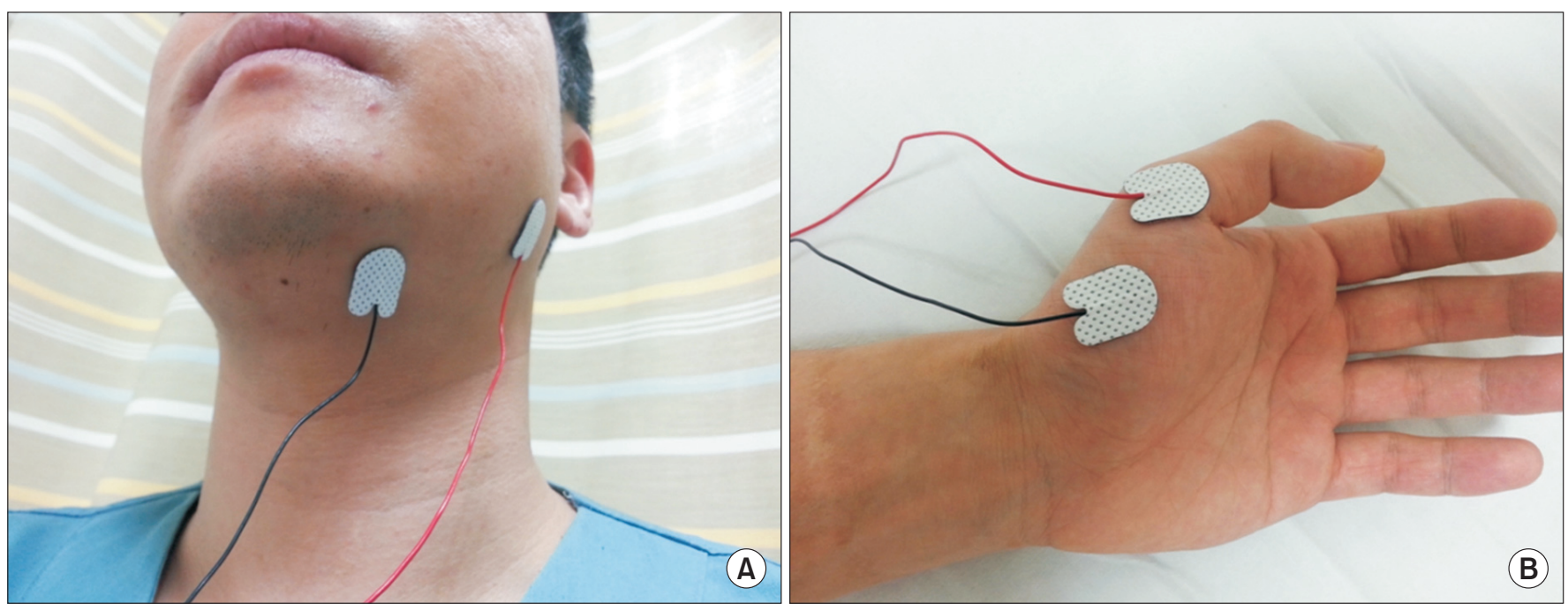

Fig. 1. Stimulation sites included the following: for group A, to stimulate a specific dysphagia site, a hot spot was obtained from motor evoked potentials (MEP) of the suprahyoid muscle (A); for group B, to stimulate an interconnected remote site related to dysphagia, a hot spot was obtained from MEP of the abductor pollicis brevis muscle (B).

All patients received the same amount of swallowing training comprised of oral and facial sensory training, oral and pharyngeal muscle training, compensatory techniques, neuromuscular electrical stimulation on pharyngeal muscles during rTMS, physical therapy, and occupational therapy.

\section{Evaluation}

To evaluate the degree of improvement in dysphagia, the Functional Dysphagia Scale (FDS), the PenetrationAspiration Scale (PAS), and the Dysphagia Outcome and Severity Scale (DOSS) were measured based on the VFSS results. FDS provides information on the oral and pharyngeal phases that can be used for quantifying dysphagia severity [21]. PAS evaluates whether the material passes through the airway during VFSS [22] (in this study, PAS for fluids was measured). DOSS documents the functional outcome of swallowing and reflects the diet status based on the objective assessment [23]. These scales were evaluated before, immediately, and 4 weeks after rTMS. VFSS was performed along with evaluations by three experienced physiatrists. To compare the cognition and functional ability among patients in each group, the Mini-Mental Status Examination (MMSE) and Korean version of the Modified Barthel Index (K-MBI) tests were conducted before, immediately after rTMS, and 4 weeks after rTMS. 
Data analysis

Repeated measures analysis of variance (ANOVA) test and Mann-Whitney U-test were used to compare and analyze the effect of rTMS according to the stimulation site. All analyses were performed with the SPSS ver. 12.0.1 (SPSS Inc., Chicago, IL, USA). The level of significance

Table 1. Demographics and disease-related characteristics of the subjects

\begin{tabular}{|lcc|}
\hline \multicolumn{1}{|c}{ Characteristic } & Group A (n=12) & Group B (n=12) \\
\hline Age (yr) & $66.1 \pm 11.2$ & $60.9 \pm 11.4$ \\
\hline Gender (male:female) & $7: 5$ & $10: 2$ \\
\hline MMSE & $18.8 \pm 2.5$ & $17.7 \pm 2.9$ \\
\hline K-MBI & $47.0 \pm 6.1$ & $50.6 \pm 7.7$ \\
\hline Time from disease onset to treatment (day) & $34.3 \pm 17.4$ & $30.9 \pm 17.2$ \\
\hline Location of lesion & & \\
\hline Cortex+subcortex & 2 & 3 \\
\hline Cortex & 4 & 4 \\
\hline Subcortex & 3 & 2 \\
\hline Brainstem & 3 & 3 \\
\hline Location of stroke (right:left) & $5: 7$ & $5: 7$ \\
\hline
\end{tabular}

Values are presented as mean \pm standard deviation or number of cases.

Group A, repetitive transcranial magnetic stimulation (rTMS) of the cortex representing the suprahyoid muscle; group B, rTMS of the cortex representing the abductor pollicis brevis; MMSE, Mini-Mental Status Examination; KMBI, Korean version of Modified Barthel Index.
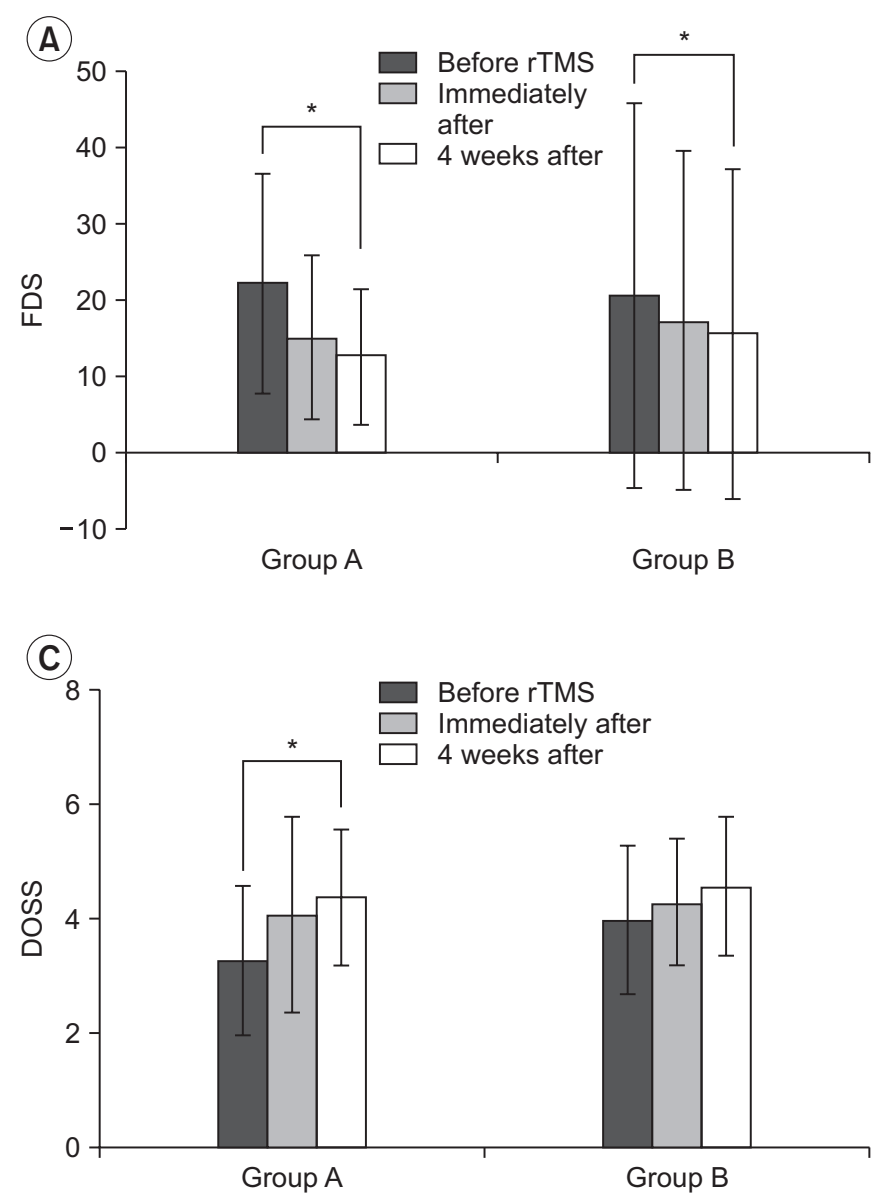

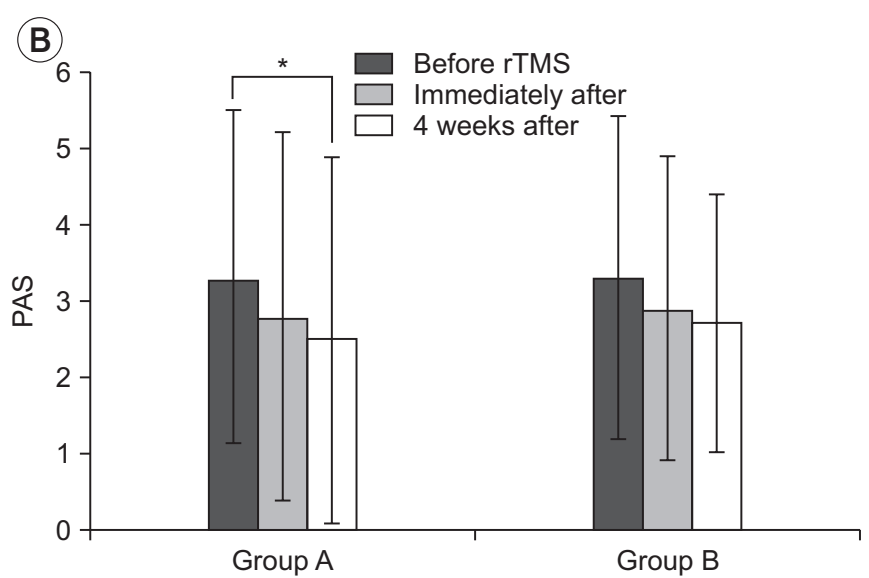

Fig. 2. In repeated measures analysis of variance test, all dysphagia scores were improved over time up to 4 weeks after rTMS in group A and only FDS score was improved in group B. There was no significant group-time interaction in all dysphagia scores. FDS, Functional Dysphagia Scale; PAS, Penetration-Aspiration Scale; DOSS, Dysphagia Outcome and Severity Scale; rTMS, repetitive transcranial magnetic stimulation; group A, rTMS over the cortex representing the suprahyoid muscle; group B, rTMS over the cortex representing the abductor pollicis brevis. ${ }^{*} \mathrm{p}<0.05$. 
was set at $\mathrm{p}<0.05$.

\section{RESULTS}

A total of 24 subjects with subacute ischemic stroke were recruited; the mean ages of patients in groups $A$ and $B$ were $66.1 \pm 11.2$ years and $60.9 \pm 11.4$ years, respectively. No significant differences in demographics or diseaserelated characteristics were observed between the two groups (Table 1).

In repeated measures ANOVA test, all dysphagia scores (FDS, PAS, DOSS) were improved over time up to 4 weeks after rTMS in group A and only the FDS score was improved in group B. There was no significant group-time interaction in all dysphagia scores (FDS, PAS, DOSS) (Fig. 2). K-MBI was improved over time up to 4 weeks after rTMS, while there was no significant group-time interaction. The MMSE score was not significantly improved over time up to 4 weeks after rTMS, and there was no significant group-time interaction (Fig. 3).

When the treatment effects from baseline to immediately after rTMS were compared between the two groups, there were no statistically significant differences in the degree of improvement in FDS or PAS scores; however, group A showed a statistically significant improvement in the DOSS score. Similarly, when the treatment effects from baseline to 4 weeks after rTMS were compared be- tween the two groups, there were no statistically significant differences in the degree of improvement in FDS or PAS scores; however, group A showed a statistically significant improvement in the DOSS score (Fig. 4).

With respect to MMSE and K-MBI, the degree of improvement from baseline to immediately after rTMS and from baseline to 4 weeks after rTMS showed no difference between the two groups (Fig. 5).

No side effects such as seizure or uncontrollable headaches were reported in either group.

\section{DISCUSSION}

This study compared dysphagia treatment effects according to the stimulation site; the cortex, responsible for innervating the suprahyoid muscle, which is related to swallowing, and the motor cortex, responsible for innervating the APB muscle. Dysphagia scores (FDS, PAS, DOSS) started to improve immediately after rTMS and continued to improve over time up to 4 weeks after rTMS. Group A showed greater improvement in the DOSS score than group $B$ in the between-group analysis.

Previous studies also performed rTMS over dysphagiarelated specific sites in post-stroke patients with dysphagia $[9,10]$. However, since sham rTMS was performed for the control group in the aforementioned study, the differences in the effects of rTMS related to the stimulation site
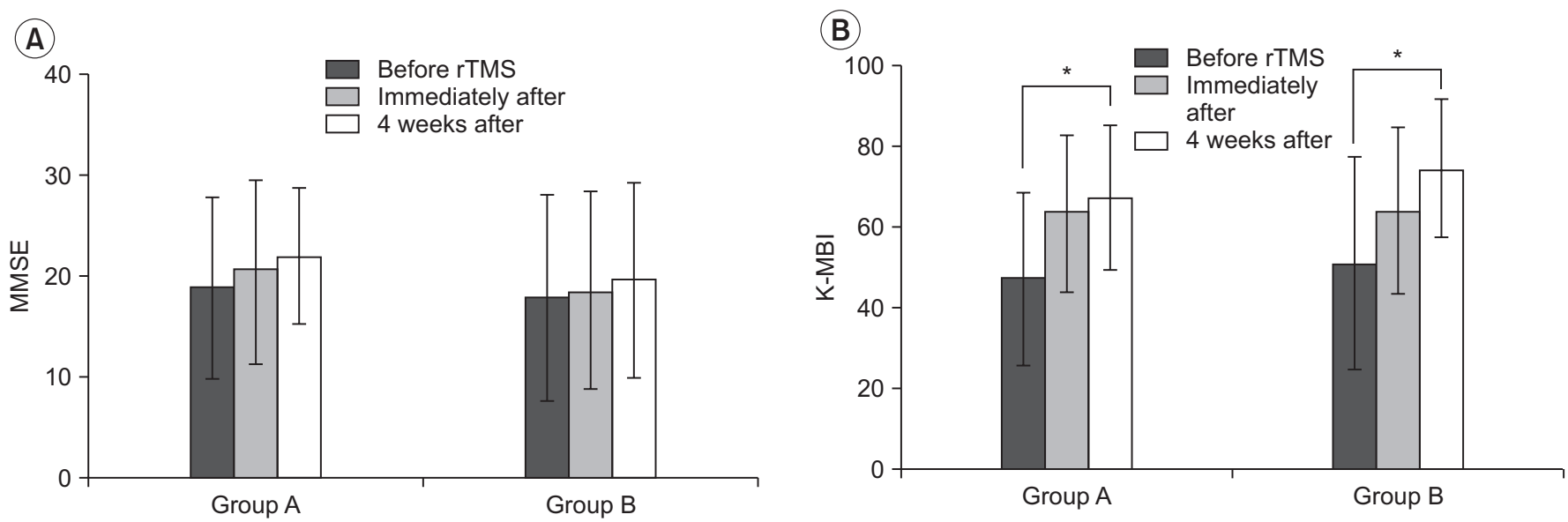

Fig. 3. In repeated measures analysis of variance test, MMSE score was not significantly improved over time up to 4 weeks after rTMS, and there was no significant group-time interaction (A). K-MBI was improved over time up to 4 weeks after rTMS, while there was no significant group-time interaction (B). MMSE, Mini-Mental Status Examination; K-MBI, Korean version of the Modified Barthel Index; rTMS, repetitive transcranial magnetic stimulation; group A, rTMS over the cortex representing the suprahyoid muscle; group B, rTMS over the cortex representing the abductor pollicis brevis. ${ }^{*} \mathrm{p}<0.05$. 

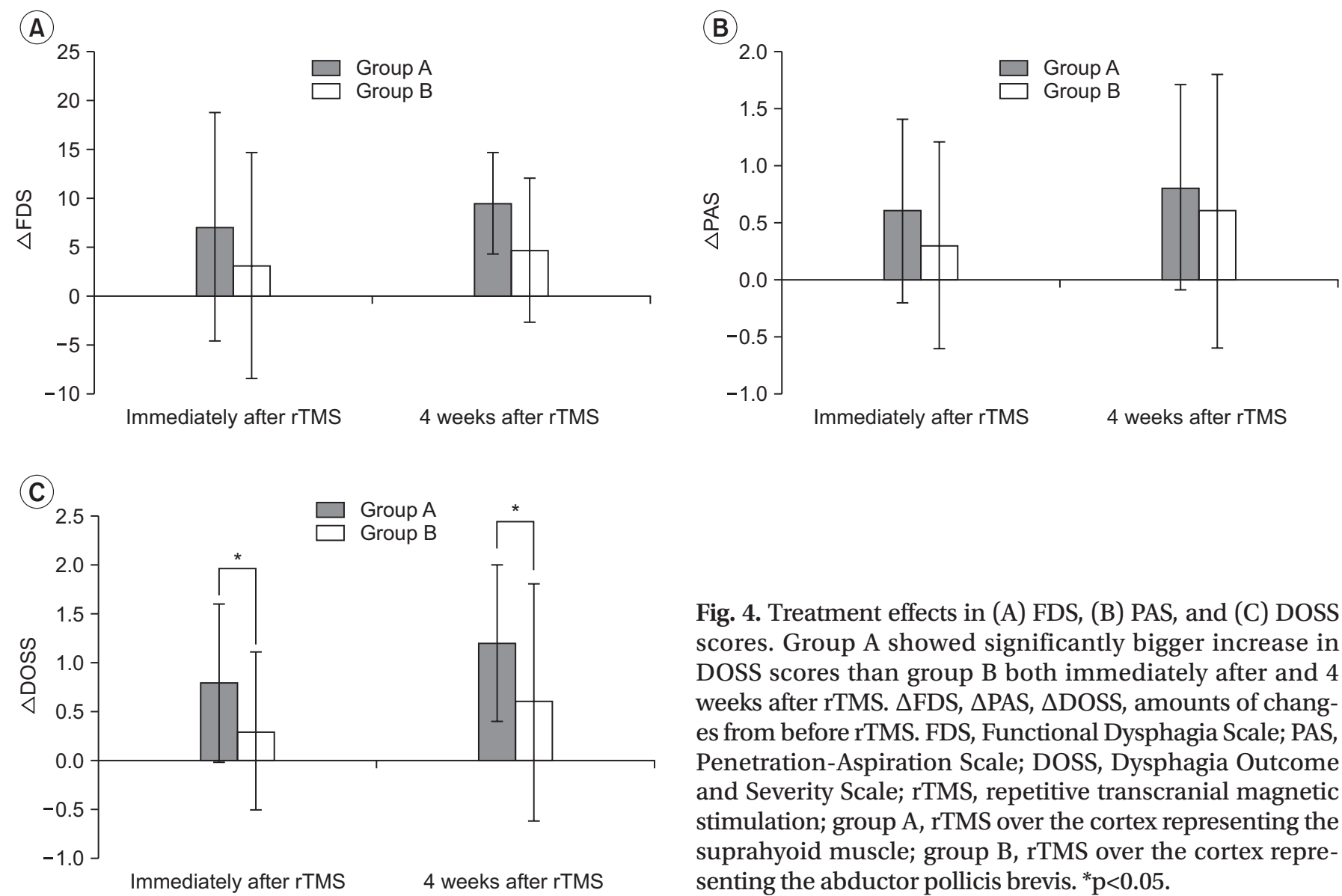

Fig. 4. Treatment effects in (A) FDS, (B) PAS, and (C) DOSS scores. Group A showed significantly bigger increase in DOSS scores than group B both immediately after and 4 weeks after rTMS. $\triangle \mathrm{FDS}, \triangle \mathrm{PAS}, \triangle \mathrm{DOSS}$, amounts of changes from before rTMS. FDS, Functional Dysphagia Scale; PAS, Penetration-Aspiration Scale; DOSS, Dysphagia Outcome and Severity Scale; rTMS, repetitive transcranial magnetic stimulation; group A, rTMS over the cortex representing the suprahyoid muscle; group B, rTMS over the cortex representing the abductor pollicis brevis. ${ }^{*} \mathrm{p}<0.05$.
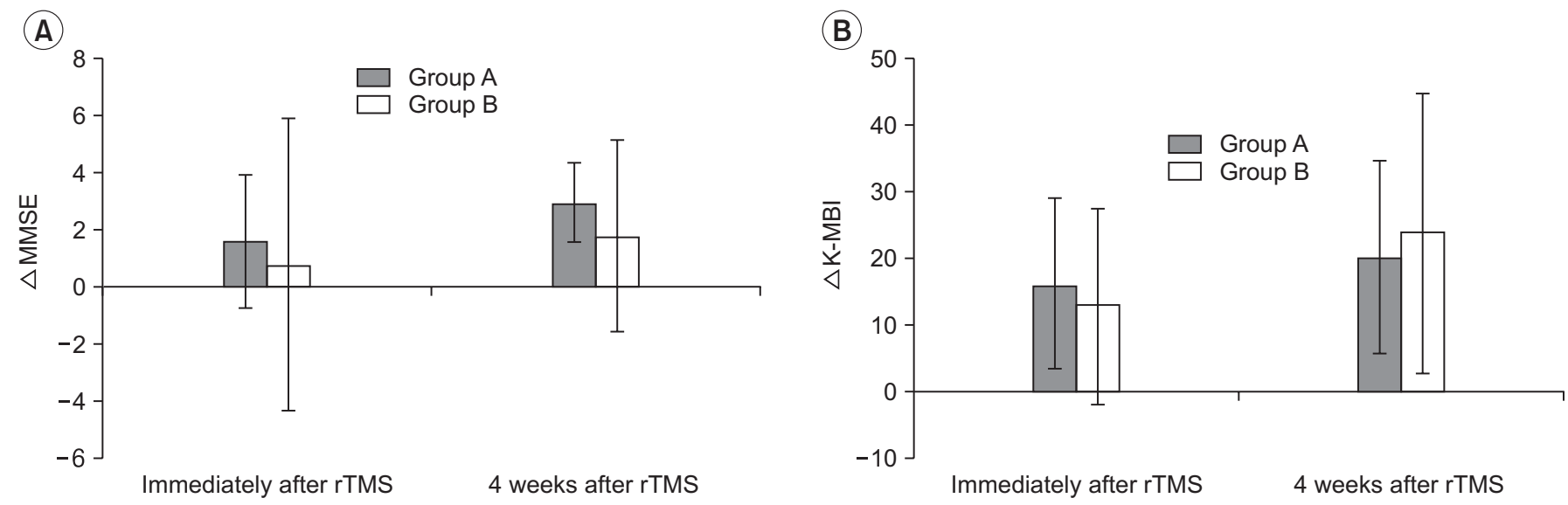

Fig. 5. When the treatment effects from baseline to immediately and 4 weeks after rTMS were compared between the two groups, no difference in improvement of the MMSE and K-MBI scores was observed (A, B). $\triangle \mathrm{MMSE}, \triangle \mathrm{K}-\mathrm{MBI}$, amount of change from before rTMS. rTMS, repetitive transcranial magnetic stimulation; Group A, rTMS over the cortex representing the suprahyoid muscle; Group B, rTMS over the cortex representing the abductor pollicis brevis; MMSE, Mini-Mental Status Examination; K-MBI, Korean version of the Modified Barthel Index.

could not be determined.

In this study, the suprahyoid muscle was selected as the specific dysphagia-related target. In post-stroke dys- phagia, the main cause of dysphagia is problems in the pharyngeal phase (decreased laryngeal elevation due to weakened muscles involved in the swallowing process) 
rather than in the oral phase [19]. Two studies reported that pharyngeal electrical stimulation to the degree of contracting muscles lowers the hyoid bone by contracting the sternohyoid and omohyoid muscles, thereby interfering with the swallowing process [24,25]. Kim and Han [26] also reported that when the suprahyoid muscle of a normal person is stimulated, the hyoid bone becomes elevated. Kim et al. [27] conducted suprahyoid muscle electrical stimulation in brain-injured patients with dysphagia and reported that this stimulation resulted in superior outcomes compared to those with conventional management.

In this study, to stimulate the interconnected sites, the cortex representing the motor hand area (APB) was stimulated. According to previous brain cortex mapping studies, there are differences in the location of the brain cortex representing the APB muscle (lateral, 6.0-6.2 cm; anterior, $0.1-0.2 \mathrm{~cm}$ from $\mathrm{Cz}$ ) and the submental (suprahyoid) muscle (lateral, $14.3 \pm 0.5 \mathrm{~cm}$; anterior, $3.9 \pm 0.5 \mathrm{~cm}$ from $\mathrm{Cz}$ ) $[20,28]$. Despite this, in this study, the group that underwent stimulation of the cortex representing the APB muscle showed improvements in swallowing function. There are two possibilities: 1) first possibility is the natural recovery of subacute stroke patients, or 2) second possibility is stimulation of the interconnected site, when the primary motor cortex is stimulated [12-17,29]. This may be because the white matter is interconnected; therefore, when the motor cortex representing the APB is stimulated, regions related to swallowing may have been stimulated.

In this study, only the DOSS score showed a statistically significant degree of improvement, which is due to the unique characteristics of each scale. Three dysphagia scales were used in this study: FDS provides information on the oral and pharyngeal phases that can be used for quantifying dysphagia severity [21]; PAS evaluates whether the material passes through the airway during VFSS [22] (in this study, PAS for fluids was measured); and DOSS documents the functional outcome of swallowing and reflects the diet status based on the objective assessment [23]. The reason why the changes in FDS scores did not show statistical significance in this study is probably that the suprahyoid muscle was selected as the dysphagiaspecific area, meaning that the pharyngeal phase was targeted. The FDS also includes an evaluation of the oral phase; thus, it is less sensitive to the degree of improvement in dysphagia. Improvement in the PAS score also did not show statistical significance; in this study, PAS evaluated only the airway invasion of fluids. Clinically, improvement in dysphagia is observed much earlier with solid foods than with fluids. For this reason, the degree of solid food-related dysphagia functional improvement was not well reflected by the PAS score. In comparison, the DOSS score reflected not only the pharyngeal phase but also the degree of improvement with regard to solid food; hence, in this study, it would have shown the highest sensitivity towards the stimulation of a dysphagiaspecific area.

This study had a few limitations. First, the sample size was small $(n=24)$. Attempts were made to minimize bias associated with the small sample size using a matched comparative study design, but the limitation remained. Second, the follow-up period was too short. Third, there was no sham rTMS control group, meaning that the improvement in dysphagia after rTMS conducted at an interconnected site could not be distinguished from that during the natural recovery of post-stroke patients in the subacute phase. Hence, future studies that address these issues are required to validate our results.

In conclusion, the results of this study showed that performing rTMS over the cortical area representing the suprahyoid muscle was more effective than performing rTMS over the cortical area representing the APB in patients with post-stroke dysphagia. In conclusion, stimulation of a specific site using rTMS according to the primary purpose or outcome can maximize its effectiveness.

\section{CONFLICT OF INTEREST}

No potential conflict of interest relevant to this article was reported.

\section{REFERENCES}

1. Martino R, Foley N, Bhogal S, Diamant N, Speechley M, Teasell R. Dysphagia after stroke: incidence, diagnosis, and pulmonary complications. Stroke 2005;36:2756-63.

2. Finestone HM, Greene-Finestone LS, Wilson ES, Teasell RW. Malnutrition in stroke patients on the rehabilitation service and at follow-up: prevalence and predictors. Arch Phys Med Rehabil 1995;76:310-6.

3. Gordon C, Hewer RL, Wade DT. Dysphagia in acute stroke. Br Med J (Clin Res Ed) 1987;295:411-4. 
4. Kidd D, Lawson J, Nesbitt R, MacMahon J. The natural history and clinical consequences of aspiration in acute stroke. QJM 1995;88:409-13.

5. Smithard DG, O'Neill PA, Parks C, Morris J. Complications and outcome after acute stroke. Does dysphagia matter? Stroke 1996;27:1200-4.

6. Sharma JC, Fletcher S, Vassallo M, Ross I. What influences outcome of stroke: pyrexia or dysphagia? Int J Clin Pract 2001;55:17-20.

7. Hamdy S, Rothwell JC, Aziz Q, Thompson DG. Organization and reorganization of human swallowing motor cortex: implications for recovery after stroke. Clin Sci (Lond) 2000;99:151-7.

8. Barwood CH, Murdoch BE. rTMS as a treatment for neurogenic communication and swallowing disorders. Acta Neurol Scand 2013;127:77-91.

9. Khedr EM, Abo-Elfetoh N, Rothwell JC. Treatment of post-stroke dysphagia with repetitive transcranial magnetic stimulation. Acta Neurol Scand 2009; 119:155-61.

10. Khedr EM, Abo-Elfetoh N. Therapeutic role of rTMS on recovery of dysphagia in patients with lateral medullary syndrome and brainstem infarction. J Neurol Neurosurg Psychiatry 2010;81:495-9.

11. Verin E, Leroi AM. Poststroke dysphagia rehabilitation by repetitive transcranial magnetic stimulation: a noncontrolled pilot study. Dysphagia 2009;24:204-10.

12. Hamdy S, Mikulis DJ, Crawley A, Xue S, Lau H, Henry $\mathrm{S}$, et al. Cortical activation during human volitional swallowing: an event-related fMRI study. Am J Physiol 1999;277(1 Pt 1):G219-25.

13. Zald DH, Pardo JV. The functional neuroanatomy of voluntary swallowing. Ann Neurol 1999;46:281-6.

14. Martin RE, Goodyear BG, Gati JS, Menon RS. Cerebral cortical representation of automatic and volitional swallowing in humans. J Neurophysiol 2001;85:938-50.

15. Mosier K, Bereznaya I. Parallel cortical networks for volitional control of swallowing in humans. Exp Brain Res 2001;140:280-9.

16. Bestmann S, Baudewig J, Siebner HR, Rothwell JC, Frahm J. Subthreshold high-frequency TMS of human primary motor cortex modulates interconnected frontal motor areas as detected by interleaved fMRI-TMS. Neuroimage 2003;20:1685-96.

17. Reithler J, Peters JC, Sack AT. Multimodal transcranial magnetic stimulation: using concurrent neuroimaging to reveal the neural network dynamics of noninvasive brain stimulation. Prog Neurobiol 2011;94:149-65.

18. Triggs WJ, McCoy KJ, Greer R, Rossi F, Bowers D, Kortenkamp S, et al. Effects of left frontal transcranial magnetic stimulation on depressed mood, cognition, and corticomotor threshold. Biol Psychiatry 1999;45:1440-6.

19. Beom J, Kim SJ, Han TR. Electrical stimulation of the suprahyoid muscles in brain-injured patients with dysphagia: a pilot study. Ann Rehabil Med 2011; 35:322-7.

20. Park SH, Song KS, Seo JH. Characteristics of motor evoked potential recording from swallowing muscles obtained by transcranial magnitic stimulation in healthy subjects. J Korean Acd Rehab Med 2009; 33:154-8.

21. Han TR, Paik NJ, Park JW. Quantifying swallowing function after stroke: a functional dysphagia scale based on videofluoroscopic studies. Arch Phys Med Rehabil 2001;82:677-82.

22. Rosenbek JC, Robbins JA, Roecker EB, Coyle JL, Wood JL. A penetration-aspiration scale. Dysphagia 1996; 11:93-8.

23. O'Neil KH, Purdy M, Falk J, Gallo L. The dysphagia outcome and severity scale. Dysphagia 1999;14:139-45.

24. Ludlow CL, Humbert I, Saxon K, Poletto C, Sonies B, Crujido L. Effects of surface electrical stimulation both at rest and during swallowing in chronic pharyngeal Dysphagia. Dysphagia 2007;22:1-10.

25. Humbert IA, Poletto CJ, Saxon KG, Kearney PR, Crujido L, Wright-Harp W, et al. The effect of surface electrical stimulation on hyolaryngeal movement in normal individuals at rest and during swallowing. J Appl Physiol (1985) 2006;101:1657-63.

26. Kim SJ, Han TR. Effect of surface electrical stimulation of suprahyoid muscles on hyolaryngeal movement. Neuromodulation 2009;12:134-40.

27. Kim JH, Lee KJ, Kim SJ. Electrical stimulation on suprahyoid muscles of the brain injury patients with dysphagia. Korean J Stroke 2007;9:134-41.

28. Jang SH, Ahn SH, Lee SJ, Ha JS, Lee H, Lee JH. The motor cortex mapping using transcranial magnetic stimulation in normal subjects. J Korean Acd Rehab Med 2000;24:219-24.

29. Bestmann S, Baudewig J, Siebner HR, Rothwell JC, Frahm J. BOLD MRI responses to repetitive TMS over human dorsal premotor cortex. Neuroimage 2005; 28:22-9. 